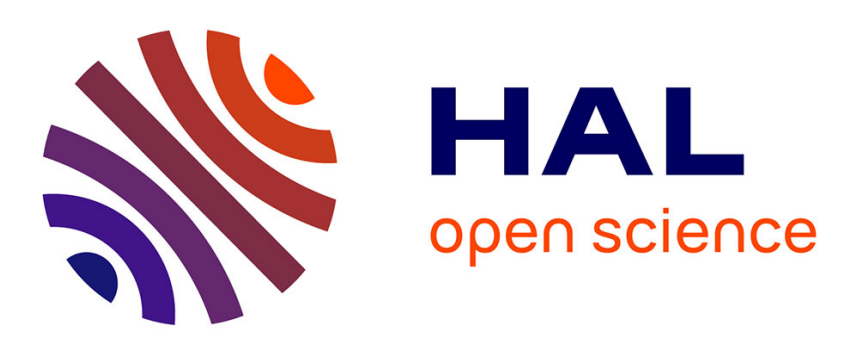

\title{
Regularised digital-level corrections for infrared image correlation
}

Ali Charbal, Stéphane Roux, François Hild, Ludovic Vincent

\section{To cite this version:}

Ali Charbal, Stéphane Roux, François Hild, Ludovic Vincent. Regularised digital-level corrections for infrared image correlation. Quantitative InfraRed Thermography Journal, 2018, 15 (2), pp.172-193. 10.1080/17686733.2018.1425955 . hal-01710068

\section{HAL Id: hal-01710068 \\ https://hal.science/hal-01710068}

Submitted on 27 Mar 2018

HAL is a multi-disciplinary open access archive for the deposit and dissemination of scientific research documents, whether they are published or not. The documents may come from teaching and research institutions in France or abroad, or from public or private research centers.
L'archive ouverte pluridisciplinaire HAL, est destinée au dépôt et à la diffusion de documents scientifiques de niveau recherche, publiés ou non, émanant des établissements d'enseignement et de recherche français ou étrangers, des laboratoires publics ou privés. 


\title{
Regularized digital level corrections for infrared image correlation
}

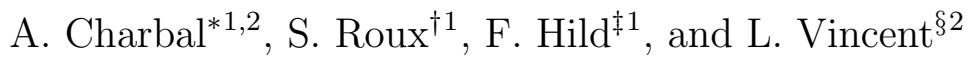 \\ ${ }^{1}$ LMT, ENS Paris-Saclay / CNRS / Université Paris-Saclay \\ 61 avenue du Président Wilson, 94235 Cachan cedex, France \\ ${ }^{2}$ DEN, Service de Recherches Métallurgiques Appliquées (SRMA) \\ CEA / Université Paris-Saclay, 91191 Gif sur Yvette cedex, France
}

February 19, 2018

\begin{abstract}
Global digital image correlation (DIC) applied to infrared images is considered for a test where very significant and localized laser heating is applied. Because of temperature changes and high gradients, digital levels are to be corrected in addition to the displacement registration. Digital level corrections and kinematics are decomposed over an unstructured mesh to account for steep gradients. A regularization strategy is used to mend ill-posedness penalizing rapid variation of corrections and displacements. High gradients motivate further softening of the regularization (i.e., reduction of the regularization length scale) based on prior estimates. The methodology is tested on different experimental cases in which DIC residuals are drastically reduced and systematic displacement errors are cut down to $10^{-2}$ pixel after gray level corrections (performed on numerical cases). Additionally, the regularization improves the convergence speed as well as the quality of the sought fields. The capability of the code to account for blur corrections is illustrated on a practical case.
\end{abstract}

Keywords: Global DIC, Gray level correction, Regularization, Thermomechanical fields

*alc517@lehigh.edu, present address: Lehigh Univ., Packard Lab, 19 Memorial D. West, Bethlehem, PA 18015, USA

${ }^{\dagger}$ stephane.roux@ens-paris-saclay.fr

${ }^{\ddagger}$ francois.hild@ens-paris-saclay.fr

§ludovic.vincent@cea.fr 


\section{Introduction}

Digital image correlation (DIC) is a powerful tool for many applications in solid mechanics [1 7]. One of the main assumptions of DIC is gray-level conservation between registered images. In many practical cases such an assumption is valid or its violation does not lead to serious consequences. Sometimes however, violation of this assumption is more detrimental. This can result for instance from lighting variations in the course of an experiment. Strategies have been designed to correct for those variations by tailoring the registration cost function to be minimized so that it becomes insensitive to simple constant, linear or affine gray level (GL) corrections where the first two are respectively termed "brightness" or "contrast" corrections. A series of DIC criteria have been proposed with different degrees of robustness (see Refs. [5,8,9 for reviews). The so-called Zero-mean Normalized Sum of Squared Differences (ZNSSD) criterion for instance is popular in local DIC measurements. For each zone of interest, gray levels are affinely transformed in such a way that each clipped image has a zero mean value and unit standard deviation [5,10]. In such a case, gray level variations become innocuous, but typically, gray level corrections are not recorded nor exploited.

Extreme cases may be met when a real image is registered with a binary reference. This situation was encountered when optical distortions of an imaging device were to be corrected for calibration purposes [11. The reference binary pattern had to be dressed by gray levels (and its blur adjusted) to be registered with a real acquisition. A specific kinematic basis issued from Refs. 12, 13 can be used. For IR cameras, the same methodology can be followed. The digital levels result from heterogeneous emissivity and temperature of the sample surface. They are strongly affected by contrast, brightness and blur variations. A shallow contrast in emissivity (e.g., between 0.8 and 0.9 ) gives a low dynamic range as compared to plain optical images. Consequently such images are more prone to noise. Moreover, they are also sensitive to artifacts such as gray level modulations due to lenses. Inhomogeneous variations over the field of view may provide spurious displacement estimates from DIC analyses and are thus to be studied in details. An Integrated DIC (I-DIC) procedure was designed for such calibration where the distortions are decomposed over a set of polynomial functions. When adapted to IR cameras, this treatment benefitted from enrichment with blur to provide satisfactory results $[11$. For other applications where gray level variations occur in a less mastered way more general strategies should be considered [14].

Filters with frequency cut-offs can be used to erase gray level variations 15. This is well understood for cases where reflections occur at specular points in the pictures [16]. The gray level corrections are identified within the DIC formalism. The same type of strategy is applied in Ref. [17] for IR frames in which the effect of a laser spot disturbed the digital level distribution on images of the deformed configuration. In a recent study [14, a more "general" method was followed consisting in decomposing the gray level corrections onto a global Finite Element (FE) representation inasmuch as for displacement fields. From a single IR camera, this formalism, termed IRIC (for InfraRed Image Correlation) 
provides both 2D kinematic and temperature fields. It was used for revealing dissipation in phase transformation bands of a shape memory alloy. In other experimental cases, temperature variations due to laser heating can reach a few hundred degrees [18,19. Even in such challenging circumstances, where gray levels (or digital levels) display huge variations, IR frames can be used to assess both temperature and displacement fields ${ }^{1}$.

In the present work, it is proposed to include gray level corrections in a global finite-element based DIC formalism [6]. Akin to the displacement field, it is proposed to account for brightness and contrast variations by fields that are decomposed onto unstructured triangular meshes. This formalism is suited to cope with regions of interest of arbitrary shape (including boundaries with acute angles or domains with inner holes). However, as finer and finer meshes are sought, ill-posedness of the DIC problem emerges, and may become limiting. In order to decouple the actual mesh fineness and the possible ill-conditioning that may result, a regularization strategy is proposed, where, in the spirit of Tikhonov regularization 20, it is proposed to add a penalization to all measured fields, including brightness, contrast and blur, when they display rapid variations. It is important though to ensure that this regularization does not interfere with expected fields [21]. In the above reference, any displacement field, which is solution to a homogeneous linear elastic problem would not be penalized.

Such corrections provide better IR frame registration and thus enable IRIC analyses to be performed even with very disturbed, i.e., gray level-wise, images. The additional soft penalization allows thermomechanical phenomena to be better captured even when small mesh sizes are considered. The use of FE meshes for the discretization of the region of interest (ROI) enables more complex topologies to be considered. Two very different examples will be shown. The first one considers IR frames during laser shocks and the second case uses the algorithm for estimating the effect of distortion (cause by lenses) on IR frames. The method proposed in Ref. 11] has shown good results using integrated DIC. However for more complex gray level distributions the used fields (i.e., set of polynomials) may not suffice. The exactly same DIC formulation, which is derived from an image analysis, between perfect and experimental frames 11, will be implemented. However the solution space of the problem will be less constrained than that designed by predefined global fields.

\section{Regularized DIC}

The proposed DIC approach is implemented in the same framework as Ref. [21], namely, for unstructured 3-noded (T3) meshes. In the following, brightness conservation is relaxed to a more general formulation of optical flow. Regularization [20] is provided by an additional cost function based on the L2 norm of

\footnotetext{
${ }^{1}$ In the context of thermal IR cameras, gray levels are more usually termed as digital levels, and after a suited calibration they are converted into temperatures under the assumption of observing a surface whose emissivity is known. In order to keep the discussion general, the "gray-level" terminology is preferred hereafter and will be used all along the remainder of the paper
} 
differential operators acting on the sought fields. The differential operator is chosen so that its kernel contains fields that are considered as physically relevant (and hence not to be penalized).

\subsection{Generalized optical flow}

The reference image is a gray level-valued scalar field, denoted $f(\boldsymbol{x})$ where $\boldsymbol{x}$ stands for the pixel coordinates. In the course of the experiment, images of the deformed state, or "deformed images" for conciseness, $g(\boldsymbol{x})$ are captured. The optical flow is in general terms the mathematical expression of the relationship between $f$ and $g$. Its most usual expression is $g(\boldsymbol{x}+\boldsymbol{u}(\boldsymbol{x}))=f(\boldsymbol{x})$, stating that the gray level is passively advected by the displacement field $\boldsymbol{u}(\boldsymbol{x})$. In this work, it is proposed to generalize this optical flow assumption to account for local gray level variations as well as (possibly heterogeneous) blur due to, say, out-of-focus effects [11]. In the absence of noise, the deformed image $g(\boldsymbol{x})$ is assumed to obey

$$
g(\boldsymbol{x}+\boldsymbol{u}(\boldsymbol{x}))=f(\boldsymbol{x})+a(\boldsymbol{x})++\sum_{i=0}^{N} b_{i}(\boldsymbol{x})\left(G_{i} * f\right)(\boldsymbol{x})
$$

where $\boldsymbol{u}(\boldsymbol{x})$ is the displacement field, $G_{i}(\boldsymbol{x})$ Gaussian kernels of different widths indexed by $i, *$ the convolution product, $a(\boldsymbol{x})$ the brightness correction, $b_{0}(\boldsymbol{x})$ and $b_{l>0}(\boldsymbol{x})$ the weights for the series of progressive blur of the reference image also accounting for contrast variations. For a more compact notation, $G_{0}(\boldsymbol{x})=$ $\delta(\boldsymbol{x})$ is chosen as the Dirac distribution and because $G_{0} * f=f$ the second term is included in the last sum. It is observed that the standard form of optical flow is recovered when $a=0$ and $b_{i}=0$ for $i \geq 0$. In the absence of blur, only $a$ and $b_{0}$ fields are needed.

Because of noise, Equation (1) cannot be fulfilled exactly, and it is proposed to measure all unknown fields, collectively denoted as $\boldsymbol{v}(\boldsymbol{x}) \equiv\left\{\boldsymbol{u}(\boldsymbol{x}), a(\boldsymbol{x}), b_{i}(\boldsymbol{x})\right\}^{\top}$ for the minimization of the quadratic norm of the residual defined as the difference between left and right members of Equation (1). Thus, the DIC cost function $\Phi_{c}^{2}$ is introduced as

$$
\Phi_{c}^{2}=\sum_{\boldsymbol{x} \in R O I}\left(a(\boldsymbol{x})+\sum_{i=0}^{N} b_{i}(\boldsymbol{x}) G_{i}(\boldsymbol{x}) * f(\boldsymbol{x})+f(\boldsymbol{x})-g(\boldsymbol{x}+\boldsymbol{u}(\boldsymbol{x}))\right)^{2}
$$

Although the minimization of Equation (2) is a highly nonlinear problem, it is proposed to solve it with a Newton scheme about the tangent problem. For this aim, the corrected deformed image $\widetilde{g}(\boldsymbol{x})$, which is based on the current determination of $\boldsymbol{v}(\boldsymbol{x})$, reads

$$
\widetilde{g}(\boldsymbol{x})=g(\boldsymbol{x}+\boldsymbol{u}(\boldsymbol{x}))-a(\boldsymbol{x})-\sum_{i=0}^{N} b_{i}(\boldsymbol{x})\left(G_{i} * f\right)(\boldsymbol{x})
$$

and the current residual

$$
\rho(\boldsymbol{x})=f(\boldsymbol{x})-\widetilde{g}(\boldsymbol{x})
$$


The unknown fields are parameterized by their discretization onto finiteelement meshes. (For rectangular ROIs, polynomial fields in an I-DIC formalism were introduced in Ref. [11] but will not be used herein.) In the following, an unstructured 2D mesh made of triangular elements with first order shape functions is selected. Hence the nodal values, $\nu_{j n}$, for node $n$, and shape function, $\chi_{n}(\boldsymbol{x})$, allow for the interpolation of any of the considered fields $v_{j}(\boldsymbol{x})$

$$
v_{j}(\boldsymbol{x})=\sum_{n} \nu_{j n} \chi_{n}(\boldsymbol{x})
$$

The same mesh is used for $a, b_{i}, u_{1}$ and $u_{2}$ the two components of the displacement field, and equivalent nodal values are introduced. Using the global notation, the shape functions are replicated over all fields to form a rectangular matrix $[\chi]$ indexed by pixels $\boldsymbol{x}$ and the column vector gathering all nodal degrees of freedom $\{\boldsymbol{\nu}\}$, so that $\boldsymbol{v}(\boldsymbol{x})=[\boldsymbol{\chi}(\boldsymbol{x})]\{\boldsymbol{\nu}\}$. The linear system in terms of the correction vector $\{\delta \boldsymbol{\nu}\}$ reads $[6$

$$
[\boldsymbol{M}]\{\delta \boldsymbol{\nu}\}=\{\boldsymbol{\beta}\}
$$

where

$$
[\boldsymbol{M}]=\sum_{\boldsymbol{x} \in R O I}[\chi(\boldsymbol{x})]^{\top}\{\boldsymbol{m}(\boldsymbol{x})\}^{\top}\{\boldsymbol{m}(\boldsymbol{x})\}[\boldsymbol{\chi}(\boldsymbol{x})]
$$

and

$$
\boldsymbol{\beta}=\sum_{\boldsymbol{x} \in R O I}[\chi(\boldsymbol{x})]^{\top}\{\boldsymbol{m}(\boldsymbol{x})\}^{\top} \rho(\boldsymbol{x})
$$

with

$$
\{\boldsymbol{m}(\boldsymbol{x})\}=\left\{f_{, x}(\boldsymbol{x}), f_{, y}(\boldsymbol{x}), 1, G_{i} * f_{0}(\boldsymbol{x})\right\}
$$

where $f_{, x}=\frac{d f}{d x}$, and $\boldsymbol{x}=(x, y)$. Once the incremental corrections $\delta \boldsymbol{v}$ are computed, the column vector $\{\boldsymbol{\nu}\}$ is updated, and the same operation is repeated until the increment is less than a chosen threshold. In all the reported results, the change in the corrections $\|\{\delta \boldsymbol{\nu}\}\|$ has to become less than $10^{-5}$. An escape condition is also added, which limits the number of iterations to 50. Those cases were encountered in practice for small mesh sizes and small regularization lengths. Their occurrence is described below but the resulting fields are not included in the discussion as the lack of convergence precludes any sound interpretation.

Designing a mesh that is coarse enough is a way to make the problem well-posed, and hopefully well-conditioned. However, it is convenient to be able to adjust the fineness of the mesh to the problem at hand, and hence ill-conditioning may occur and sensitivities to noise. An alternative route is provided by incorporating an extraneous requirement that penalizes rapid spatial variations of the correction fields $\boldsymbol{v}$. This strategy (i.e., regularization) is developed below. 


\subsection{Bulk regularization}

Following the approaches developed in Refs. 21, 22, a natural way to penalize displacement fields that are physically unrealistic is to express in mathematical terms what is expected from those fields, say, in regions where DIC information would be lacking. The most elementary answer is to refer to a reference elastic medium. In general, with no further information, it may be assumed that no body forces are present, and hence the balance condition, $\operatorname{div}(\boldsymbol{\sigma})=\mathbf{0}$, is expected to hold (where $\boldsymbol{\sigma}$ stands for the Cauchy stress tensor). Assuming a known elastic law with Hooke's tensor $\boldsymbol{C}$, the displacement field $\boldsymbol{u}$ should obey the following differential equation $\boldsymbol{\nabla} \cdot(\boldsymbol{C}: \boldsymbol{\nabla} \otimes \boldsymbol{u}(\boldsymbol{x}))=\mathbf{0}$. In the above mentioned finite element framework, this differential equation takes the discrete form

$$
\left[\boldsymbol{K}_{e}\right]\{\boldsymbol{u}\}=\{\mathbf{0}\}
$$

for all inner nodes, where $\{\boldsymbol{u}\}$ is the column vector gathering all nodal displacements, and $\left[\boldsymbol{K}_{e}\right]$ the classical stiffness matrix. The regularization strategy proposed in Refs. 22, 22 consists of adding a cost function proportional to the L2 norm of the left hand side of Equation 10. . For compactness, it is convenient to introduce a diagonal matrix $[\boldsymbol{D}]$ such that $D_{i j}=1$ only if $i=j$ and $i$ refers to inner nodes (or a traction-free boundary node), and $D_{i j}=0$ otherwise. The elastic regularization cost function is defined as

$$
\Phi_{e}^{2}=\{\boldsymbol{u}\}^{\top}\left[\boldsymbol{K}_{e}\right][\boldsymbol{D}]\left[\boldsymbol{K}_{e}\right]\{\boldsymbol{u}\}
$$

where the symmetry of $\left[\boldsymbol{K}_{e}\right]$ has been used.

In a similar spirit, the correction fields are on a general ground linearly related to a temperature field, $T(\boldsymbol{x})$, and the latter, in the absence of source terms, and for linear Fourier's law with a uniform isotropic conductivity, obeys $\Delta T(\boldsymbol{x})=0$. A discretized version of this equation over the same mesh as used previously reads

$$
\left[\boldsymbol{K}_{t}\right]\{\boldsymbol{T}\}=\{\mathbf{0}\}
$$

for all interior nodes, where $\{\boldsymbol{T}\}$ is the column vector gathering all nodal temperatures. Hence, the thermal regularization cost function is defined as

$$
\Phi_{t}^{2}=\{\boldsymbol{T}\}^{\top}\left[\boldsymbol{K}_{t}\right][\boldsymbol{D}]\left[\boldsymbol{K}_{t}\right]\{\boldsymbol{T}\}
$$

where the symmetry of $\left[\boldsymbol{K}_{t}\right]$ has been exploited. This regularization term is to be used substituting the nodal temperatures by either nodal vectors $\{\boldsymbol{a}\}$ or $\left\{\boldsymbol{b}_{i}\right\}$. The same $[\boldsymbol{D}]$ matrix is used herein, assuming that mechanically free edges are also devoid of external heat flux. It is straightforward to generalize this treatment to different $\mid \boldsymbol{D}]$ matrices if needed.

Let us stress that the kernel of the cost function is the set of all harmonic fields (i.e., obeying $\Delta T=0$ ), and hence any such field will not be affected by the penalty term $\Phi_{t}^{2}$.

\subsection{Boundary regularization}

The above bulk regularizations exploit models that may at least be a reasonable approximation for the measured quantities. They assume that no heat source 
nor external loads are applied inside the studied region of interest. As earlier mentioned, this property also includes free edges that can be considered as interior nodes. For non-free edges, no first-principle information is available.

What is proposed herein is to be consistent with the previous approach and introduce quadratic cost functions on these boundary conditions, $\Phi_{e b}^{2}$ and $\Phi_{t b}^{2}$ respectively for the displacement and gray level correction degrees of freedom. One may require that they be quadratic norms of differential operators of a similar order as for the bulk, 2 , so that they remain unaffected by any spatially linear transforms. Moreover, for the elastic part, one may require that rotational invariance is obeyed, which will select a differential operator that can be interpreted as resulting from a physically sound elastic surface model [21].

When the same approach is applied to the heat transfer side, it automatically selects a surface model such that the quadratic norm of $\partial_{\|}^{2} T$ is minimized (here $\partial_{\|}$denotes the gradient component along the boundary). The consequence of considering this boundary regularization term is to smooth out heat fluxes that would flow across the considered boundary.

\subsection{Regularization weights}

In order to normalize all the previous cost functions, it is convenient to scale them by the value taken for a reference correction field $\boldsymbol{v}_{r e f}(\boldsymbol{x})=\boldsymbol{v}_{0} \exp \left(i \boldsymbol{k}_{0} \cdot \boldsymbol{x}\right)$, which can be characterized by an intrinsic length scale $\ell_{0}=2 \pi /\left|\boldsymbol{k}_{0}\right|$. Additionally, it is chosen to prescribe $\left|\boldsymbol{v}_{0}\right|=1$. Let us introduce,

$$
\widehat{\Phi}_{X}^{2}(\boldsymbol{v}) \equiv \frac{\Phi_{X}^{2}(\boldsymbol{v})}{\Phi_{X}^{2}\left(\boldsymbol{v}_{r e f}\right)}
$$

where the index $X$ is either the DIC, $X=c$, or the elastic $X=e$ or thermal $X=t$ regularization cost functions, bulk or boundary terms alike.

The dependence of $\widehat{\Phi}_{X}$ with the intrinsic length scale $\ell_{0}$ or "wave number" $\left|\boldsymbol{k}_{0}\right|$ is studied. The DIC functional is independent of the wave number, but the two proposed regularization functionals, are quadratic forms based on a second-order differential operator, and hence they are homogeneous functions in $\left|\boldsymbol{k}_{0}\right|$ of degree 4. As normalized, all of these functionals are valued 1 for $\boldsymbol{v}_{r e f}$. If a similar test field as $\boldsymbol{v}_{r e f}$ is constructed with a different wavelength, $\ell$, then $\widehat{\Phi}_{e}^{2}(\boldsymbol{v})=\widehat{\Phi}_{t}^{2}(\boldsymbol{v})=\left(\ell / \ell_{0}\right)^{4}$ and $\widehat{\Phi}_{c}^{2}(\boldsymbol{v})=1$. It means that over large scales (i.e., larger than $\ell_{0}$ ), DIC is dominant and regularization plays virtually no role. Conversely, at small scales, regularization takes over and the resulting fields mostly obey the regularization equation and DIC plays a minor role. This means that a low pass filter is defined for DIC, where high frequencies are accounted for by fields that locally obey a differential equation rather than being a mathematically convenient function. This allows for much more flexibility than modifying a mesh.

The advantage of having kept the different fields in separate functionals is that, different cross-over length scales can be assigned to each of them, be they relative to the bulk or the edges of the ROI. This being decided, the global 
regularized functional reads

$$
\begin{aligned}
\Phi_{r e g}^{2}(\{\boldsymbol{\nu}\}) & \equiv\{\boldsymbol{\nu}\}^{\top}[\boldsymbol{L}]\{\boldsymbol{\nu}\} \\
& =\left(\frac{\ell_{\boldsymbol{u}}}{\ell_{0}}\right)^{4} \widehat{\Phi}_{e}^{2}(\{\boldsymbol{u}\})+\left(\frac{\ell_{a}}{\ell_{0}}\right)^{4} \widehat{\Phi}_{t}^{2}(\{\boldsymbol{a}\})+\sum_{i}\left(\frac{\ell_{b i}}{\ell_{0}}\right)^{4} \widehat{\Phi}_{t}^{2}\left(\left\{\boldsymbol{b}_{i}\right\}\right) \\
& +\left(\frac{\bar{\ell}_{\boldsymbol{u}}}{\ell_{0}}\right)^{4} \widehat{\Phi}_{e b}^{2}(\{\boldsymbol{u}\})+\left(\frac{\bar{\ell}_{a}}{\ell_{0}}\right)^{4} \widehat{\Phi}_{t b}^{2}(\{\boldsymbol{a}\}) \\
& +\sum_{i}\left(\frac{\bar{\ell}_{b_{i}}}{\ell_{0}}\right)^{4} \widehat{\Phi}_{t b}^{2}\left(\left\{\boldsymbol{b}_{i}\right\}\right)
\end{aligned}
$$

where the matrix $[\boldsymbol{L}]$ is block diagonal, without coupling between the different fields $\boldsymbol{u}, a$ and $b_{i}$, and $\ell_{\boldsymbol{u}}, \ell_{a}, \ell_{b_{i}}, \bar{\ell}_{\boldsymbol{u}}, \bar{\ell}_{a}, \bar{\ell}_{b_{i}}$ all the regularization lengths, which are expressed in pixels with the chosen normalization.

\subsection{Implementation}

The implementation is similar to Ref. 21 i.e., a Newton iterative scheme is used to solve the nonlinear problem of minimizing $\Phi_{\text {tot }}^{2}(\{\boldsymbol{\nu}\})=\widehat{\Phi}_{c}^{2}(\{\boldsymbol{\nu}\})+\Phi_{\text {reg }}^{2}(\{\boldsymbol{\nu}\})$. The incremental corrections that were introduced in Equation (6) now satisfy a new linear system of equations

$$
([\boldsymbol{M}]+[\boldsymbol{L}])\{\delta \boldsymbol{\nu}\}=\{\boldsymbol{\beta}\}-[\boldsymbol{L}]\{\tilde{\nu}\}
$$

where $\{\tilde{\nu}\}$ is the current estimate of the nodal vector $\{\boldsymbol{\nu}\}$. It is worth noting that at each iteration the corrected image $\tilde{g}$ and the residual field $\rho$ have to be recomputed for each pixel $\boldsymbol{x}$ belonging to the region of interest. A bi-cubic interpolation is used for the gray levels. Conversely, the Hessian of the linearized function $[\boldsymbol{M}]$ and the regularization matrix $[\boldsymbol{L}]$ are computed once for all before the iterative scheme is used.

\subsection{Remark on temporal regularization}

In the present work, only spatial regularization is aimed for. One of the reasons is that the vast majority of DIC analyses are based upon so-called incremental calculations in which only two images are registered, namely, one in the reference configuration and the other one in the deformed configuration. Few cases have been analyzed by explicitly using temporal regularization. Broggiato et al. 23 proposed to use 5 consecutive pictures for global DIC analyses and a restricted (parabolic) time change of the displacement field. This multi-frame procedure leads to a smoother time evolution of strain rates. It was used to have a more precise evaluation of the strain rates in the central frame (i.e., local in time). The combined use of space and time kinematic bases allows the spatial resolution to be decreased by increasing the temporal resolution to achieve similar or even lower uncertainty levels 24. Last, for IR pictures, spatiotemporal regularizations based upon Karhunen-Loeve decomposition are yet another alternative route [25]. 


\section{Synthetic test case}

A synthetic test case is first considered to validate the proposed procedure and assess the resolution that can be expected for an ideal situation where no model error is present. Image pairs are constructed so that the exact transformation is known. However, in order to be representative of a real experiment, the test case was built from a real reference image and the deformed image is created from what can be modelled as a real experimental case, here a laser shock [26]. The laser beam impacts a circular domain on the surface of a sample and the thermal shock induces an expansion of the hot region in addition to large gray level modulations. The deformed image is computed to match exactly the assumed transformation, so that a direct comparison can be computed. Figure 1 shows the image pair. In this section, no blur is considered, so that $b_{i}$ reduces to $b_{0}$ and is thus denoted as $b$ for the sake of conciseness.

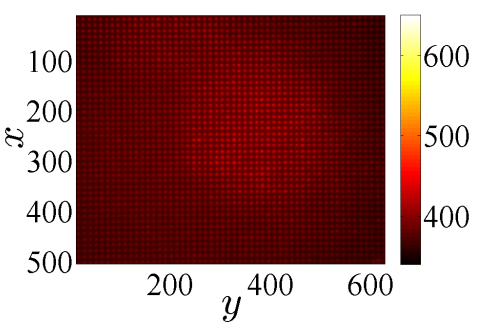

(a)

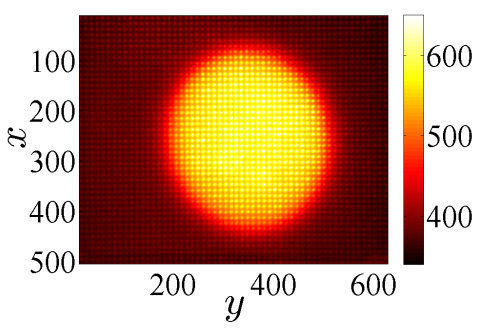

(b)

Figure 1: (a) Reference image and (b) synthetically deformed image mimicking laser illumination and thermal expansion of the hot region

\subsection{Artificial laser shock generation}

The applied fields $u_{x}, u_{y}, a$ and $b$ are illustrated in Figure 2, Let us stress that no additional noise is introduced in the computed deformed image except that induced by gray level interpolation. 


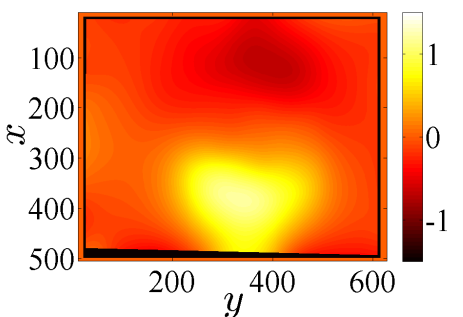

(a)

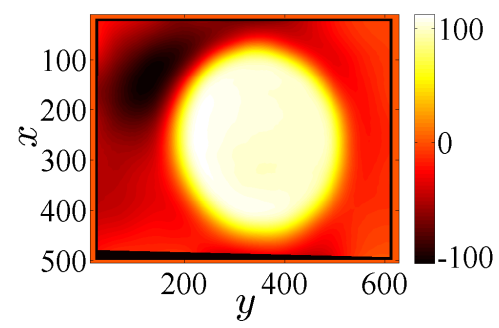

(c)

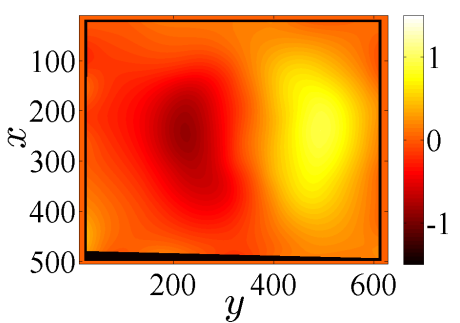

(b)

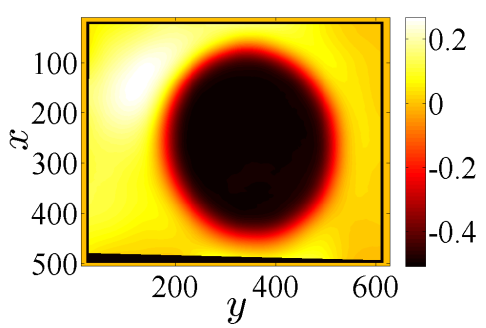

(d)

Figure 2: (a) $x$ and (b) $y$ components of the prescribed displacement field (expressed in pixel). Gray level brightness (c) and contrast (d) correction fields used to generate the artificial test case

In the following, the residual $\left\langle(\widetilde{g}-f)^{2}\right\rangle^{1 / 2}$ is normalized after correction by the reference dynamic range, $\max (f)-\min (f)$, and is denoted as $\omega$. It will be used to evaluate the quality of the computed solution with a single number. For the present test case, the computation of $\omega$ prior to any correction, i.e., using directly $g$ instead of $\widetilde{g}$, amounts to $85 \%$. This level is mostly due to the large variations of the gray levels (Figure 2(c)), while the mismatch in position has a much smaller weight.

The challenge of this test case is the very sharp gradient in the gray level corrections (Figure 2(c-d)). In order to be able to resolve it, it is necessary (although not sufficient) to use a very fine mesh. An unstructured mesh composed of T3 (three-node triangular elements) was used with a typical edge length of 4 pixels. In the following, the edge regularization is kept identical to the bulk one for each field, i.e., $\ell_{X}=\bar{\ell}_{X}$ for all $X$. The influence of the regularization length is first described and the effect of the mesh size will be reported in the following section.

\subsection{Influence of regularization length}

The effect of the regularization length scale is now studied systematically, through different kinematic, $\ell_{e}$, and gray level corrections, $\ell_{a}=\ell_{b}$, regularization lengths and the main results are shown in Figure 3. First, Figure 3(a) 
shows that regularization helps to speed up convergence. As the regularization lengths decrease, convergence becomes slower, and finally DIC fails to converge. Because of the very small size of the mesh, conditioning becomes poorer and finally ill-posed as the regularization lengths $\ell$ decrease. For a mesh size of 4 pixels, a small gray level regularization length, $\ell_{a}<20$ pixels does not yield a converged result (for the chosen criterion), and this irrespective of the elastic regularization length $\ell_{e}$ ranging from 8 to 25 pixels. This conclusion is consistent with the observation that the gray level correction is dominant in this test case.

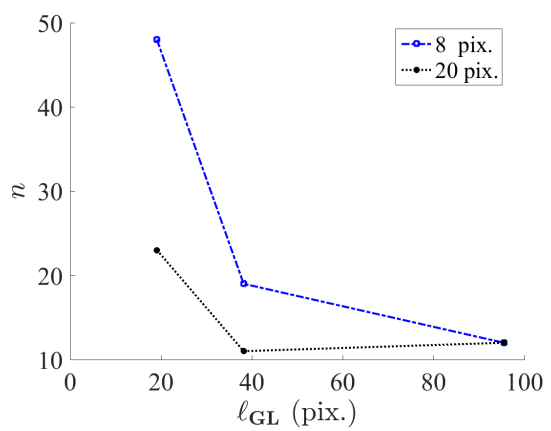

(a)

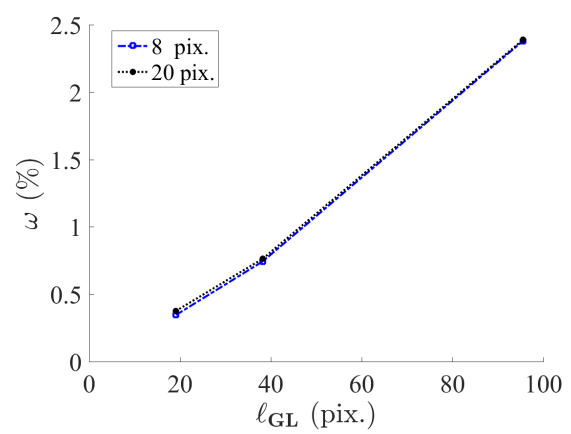

(b)

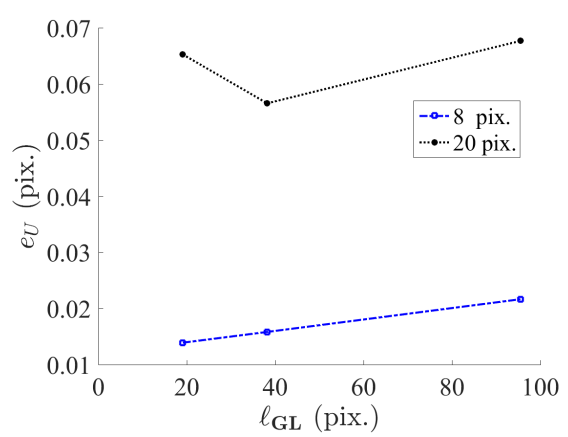

(c)

Figure 3: (a) Number $n$ of iterations at convergence. (b) Normalized residual $\omega$ and (c) RMS displacement error $e_{U}$ as functions of the regularization length $\ell_{G L}=\ell_{a}=\ell_{b}$ for different kinematic regularization lengths $\ell_{e}$. The mesh size is 4 pixels

However, convergence does not mean that the result will be satisfactory nor considered as such. Figure 3(b) shows that the normalized residual $\omega$ at convergence increases with the regularization lengths for large values. This observation comes from the fact that the regularizing models do not involve any source and hence they cannot reproduce the fields shown in Figure 2, Large regularization lengths $\ell$ mean that sharp transitions are smeared over larger distances, and hence induce larger discrepancies between $\widetilde{g}$ and $f$. A very progressive decrease in residuals is seen down to a value of about $\ell_{a}=\ell_{b}=10$ pixels, below which the behavior becomes more erratic, and the residual increases. This is due to a lack of convergence as earlier reported. For small regularization lengths $\ell$, spu- 
rious minima become accessible and may trap the minimization scheme even if the residual score is higher than the global minimum. Hence the limitation is a stability issue, and this constitutes a further motivation for using a regularization strategy. This result points out to using an $\ell$ value about 20 pixels for good convergence and reasonable convergence speed.

It was earlier mentioned that most of the residual score was due to gray level corrections in the initial state, and this property also survives at convergence. Hence, it may righteously be deduced that the residual is not informative on the kinematic registration. Figure 3(c) shows precisely the change of the RMS error in displacements with the various regularization lengths $\ell$. It is observed that the minimum is reached for $\ell_{a}=\ell_{b}=8$ pixels and $\ell_{a}=\ell_{b}=10$ pixels, consistently with the value of the global residual. Yet, the shape of the curves is different. As soon as convergence becomes dubious, error and residual increase very fast, again signaling stability (i.e., conditioning) issues.

The slight difference in optimal length scale suggests to tune separately the different regularization lengths. There is no necessity to keep $\ell_{e}=\ell_{a}=\ell_{b}$ and hence they may be adjusted separately to reach the best solution.

\subsection{Influence of mesh size}

In this section the kinematic regularization length scale is set to $\ell_{e}=20$ pixels, and the mesh size will explore values in the range from 8 to 64 pixels so as to favor convergence. Figure 4 shows the same plots as in Figure 3 but for different mesh sizes. The previously observed acceleration of convergence for larger $\ell_{a}=\ell_{b}$ lengths is observed to be less pronounced for larger mesh sizes (Figure 4(a)) because a larger mesh size limits or cancels out the high frequency modes that are poorly conditioned. It is seen that for a mesh size greater than or equal to 8 pixels, convergence is achieved for all gray level regularization lengths down to 0 (i.e., no gray level regularization at all). The discretization of fields over a mesh is by itself a regularization from the limitation of the measured fields to a set of (here) piecewise linear and continuous shape functions. This, however, does not say much about the quality of the estimated fields. It is also observed that for a very coarse mesh (i.e., 64 pixels) the trend becomes opposite. 


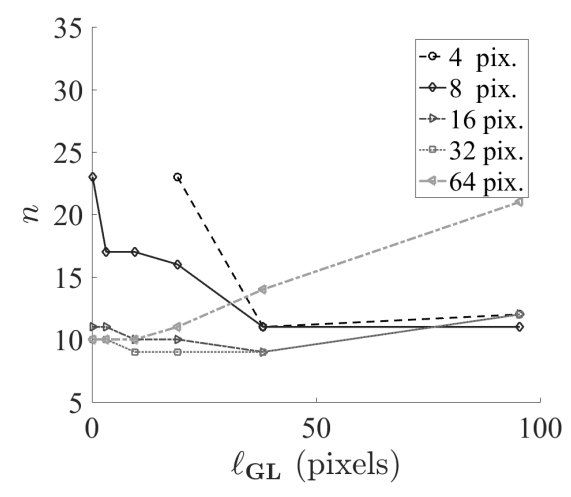

(a)

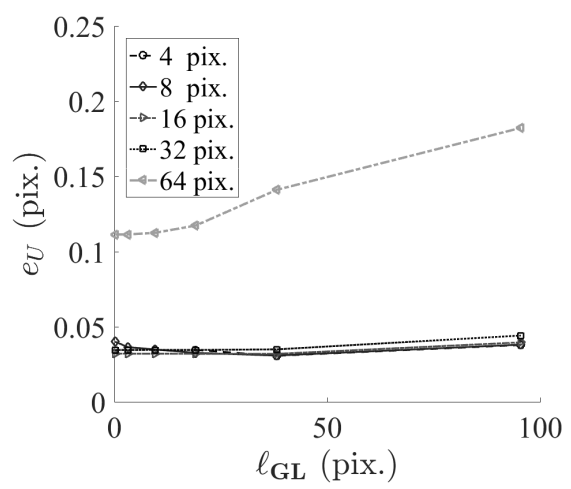

(c)

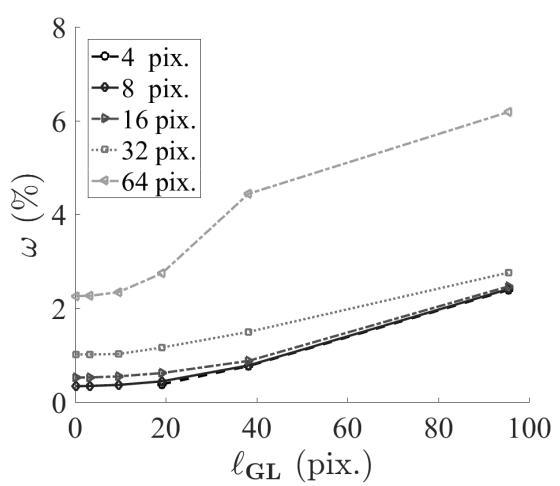

(b)

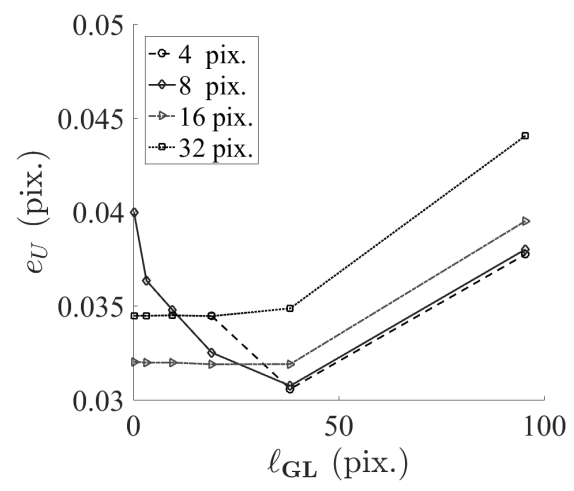

(d)

Figure 4: (a) Number of iterations at convergence. (b) Normalized residuals, and (c-d) RMS displacement error $e_{U}$ as functions of the gray level regularization length for different mesh sizes. For all plots, the kinematic regularization length is set to $\ell_{e}=20$ pixels

Figure 4(b) shows that such a large (i.e., 64 pixel) mesh size induces large systematic errors for all values of the regularization length. For finer meshes, a unique curve is progressively approached, where regularization provides the only relevant scale. Therefore, from this analysis it is deduced that gray level regularization does not induce biases to the solution only for $\ell_{a}=\ell_{b}<16$ pixels and for a mesh size of 8 pixels or less. Yet if smaller scales are chosen, lack of convergence (or at least slow convergence) may appear.

Figure 4(c) shows the root mean square (RMS) displacement error. It confirms that a very coarse mesh (i.e., 64 pixel elements) is unadapted to the test case, and it induces a large systematic error. For smaller mesh sizes, the effect on the displacement field is modest. This is due to the fact that the displacement field is smoother than the gray level corrections and may be well described even for a mesh size of 32 pixels. Moreover for this study the kinematic regularization is kept equal to $\ell_{e}=20$ pixels. Thus it is observed that using a too strong gray level regularization such as $\ell_{a}=\ell_{b}=95$ pixels, whose quality is poor as judged from the global residual, has a very limited influence on the displacement field. 
In order to illustrate the meaning of the global residual, residual maps are shown in Figure 5 for mesh sizes of 4 and 32 pixels. Their global residual score amounts to $\omega=0.3 \%$ and $1.1 \%$, respectively. For the coarser mesh, residuals concentrate over the region where gray level gradients are the largest.

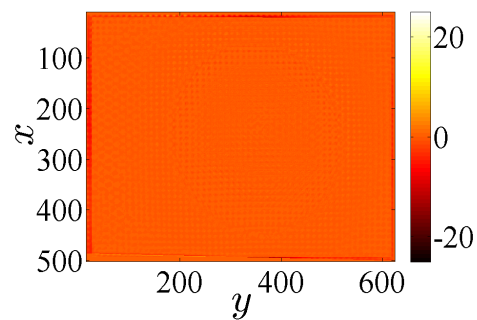

(a)

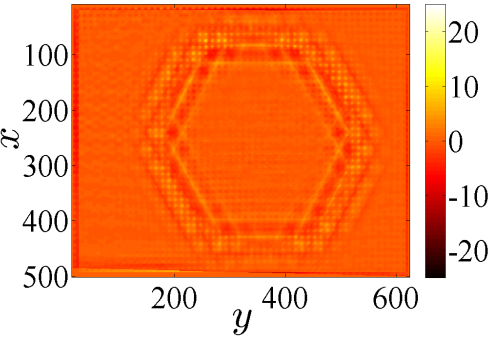

(b)

Figure 5: DIC residual maps (expressed in gray levels) for a mesh size of (a) 4 and (b) 32 pixels with gray level and kinematic regularization lengths $\ell_{a}=$ $\ell_{b}=\ell=20$ pixels

To highlight the parallel in terms of regularization, Figure 6 shows the DIC residual maps for regularization lengths $\ell_{a}=\ell_{b}=95$ and $\ell_{a}=\ell_{b}=20$ pixels. A large regularization length gives rise to a large model error (as observed with $\ell_{a}=\ell_{b}=95$ pixels from Figure 4(b)). The residual field signals that the constrains on the gray level corrections are too stringent to account for the real ones, whereas $\ell_{a}=\ell_{b}=20$ pixels succeeds in bringing the residual down almost to 0 over the same color scale.

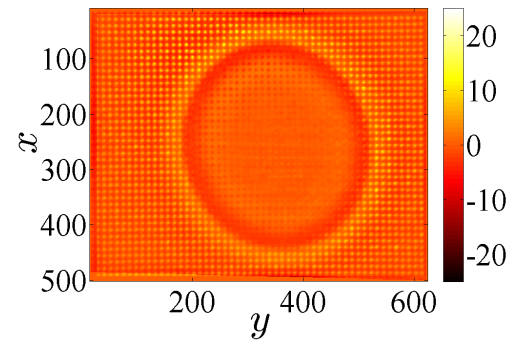

(a)

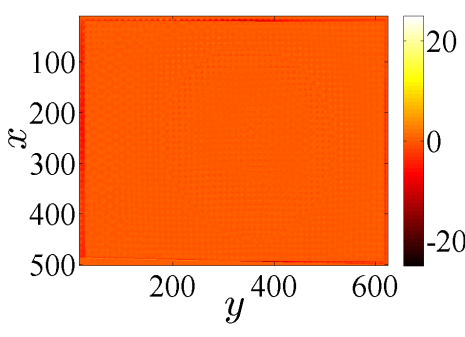

(b)

Figure 6: DIC residuals maps (expressed in gray levels) obtained with a mesh size of 4 pixels and regularization lengths $\ell_{a}=\ell_{b}=600$ pixels (a) and $\ell_{a}=$ $\ell_{b}=120$ pixels $(b)$

\subsection{Spatially adapted regularization}

In the previous section it has been shown that the regularization length has to be set in order to capture the steep gradients in gray levels on the edge of the 
laser spot. However, away from this ring, the sought fields are very smooth and could be captured with a large regularization length with the benefit of fast convergence and low sensitivity to noise due to the better conditioning of the incremental linear system to solve at each iteration. Thus, it is natural to design a strategy whereby the regularization length would no longer be uniform, but rather spatially adapted to the field. The idea is to relax the regularization constraint where a feature (here a large gradient) is detected and is penalized by regularization. Let us introduce a weight varying between 0 and 1 , such that

$$
d=d_{0}+\left(1-d_{0}\right) \exp \left(-\left\|\nabla\left(G_{1} * g_{0}\right)\right\|^{2} / \alpha^{2}\right)
$$

This parameter (whose complement to 1 can be seen as a kind of damage parameter [27]) varies from $d=1$ when $\left\|\boldsymbol{\nabla}\left(G_{1} * g_{0}\right)\right\|=0$ to $d_{0}$ for very large gradients. $\alpha$ sets the magnitude of the gradient where $d$ drops from 1 to $d_{0}$. The field of $d$ can be computed element-wise once $\{\boldsymbol{a}\}$ has been evaluated. Using the above estimate of the $d$ field, one may revisit the regularization operator. Only the gray level corrections are weighted as it was seen that the smoother kinematics was well captured with a uniform regularization. The stiffness matrix $\left[\boldsymbol{K}_{t}\right]$ is the assembly over elements $j$ of elementary matrices $\left[\boldsymbol{K}_{t}^{(j)}\right]$. Thus it is proposed to weight each elementary matrix by $d_{j}$ so that

$$
\left[\overline{\boldsymbol{K}}_{t}\right] \equiv \sum_{j} d_{j}\left[\boldsymbol{K}_{t}^{(j)}\right]
$$

From those relaxed stiffness matrices, the regularization matrix $[\boldsymbol{L}]$ is reassembled. The $d_{0}$ parameter defines the minimum weight to be given to the regularization in order not to generate ill-conditioning. It is arbitrarily set to $d_{0}=0.1$ in the following. It is to be noted that as written, the regularization becomes a nonlinear problem, because $d$ is computed from $\{\boldsymbol{a}\}$, which itself depends on the solution to the regularized problem. It is proposed to start the process with a large regularization length smoothing out the steep gradients, and progressively lower the weight given to those elements where the gradients are the largest (i.e., they will be subjected to a weaker regularization operator), and hence gradients are expected to concentrate over a small support. It is also very important to choose $\alpha$ appropriately. Here it is suggested to study the histogram and spatial distribution of $\left\|\nabla\left(G_{1} * g_{0}\right)\right\|$ in order to select $\alpha$ such that it captures gradients in the beam spot edge in a conservative fashion.

Figure 7 shows the results obtained for a mesh size of 16 pixels, a kinematic regularization length of 20 pixels, and initial lengths $\ell_{a}=\ell_{b}=38$ pixels. The initial brightness field shown in Figure 7(a) is too smooth because of the choice of $\ell_{a}$ that prevents sharp boundaries. From the deformed image gradient, a weighting field $d$ is computed as shown in Figure 7(b). The resulting brightness and residual fields are displayed in Figures $7(\mathrm{c}-\mathrm{d})$. It is observed that the prescribed brightness field is much better captured at the beam spot edge, and the residuals are lowered, close to the minimum value obtained with a much finer regularization length. 


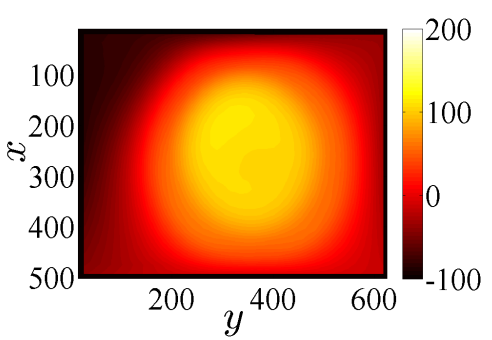

(a)

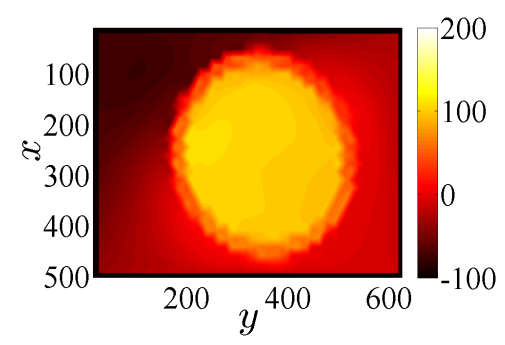

(c)

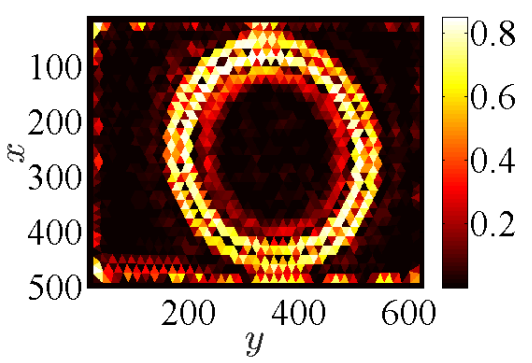

(b)

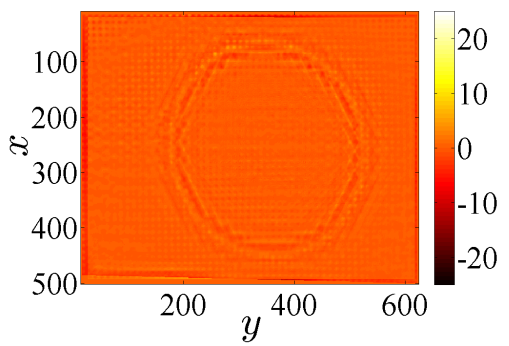

(d)

Figure 7: (a) Initial brightness field $a$ (expressed in gray levels) estimated with an identical regularization length of $\ell=\ell_{a}=\ell_{b}=38$ pixels. (b) Weighting field $d$ and (c) resulting brightness field with a relaxed weight at the sharp gradients and $(\mathrm{d})$ residual field. The mesh size is 16 pixels

The adaption of the regularization length is now tested over different mesh sizes. Figure 8 shows the dimensionless residual $\omega$ for different mesh sizes and initial regularization length. 


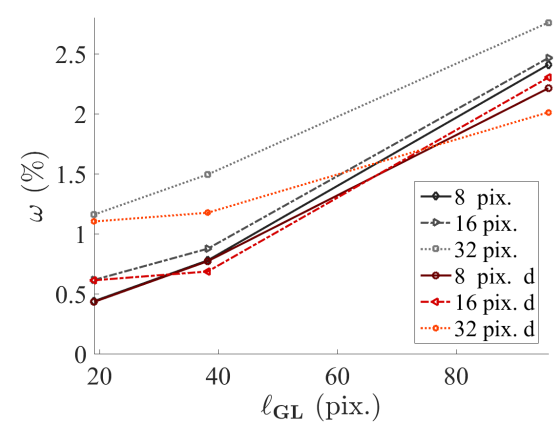

Figure 8: Normalized DIC residuals $\omega$ obtained with the adapted regularization strategy for various mesh sizes and initial regularization lengths $\ell=\ell_{a}=\ell_{b}$ (in the legends with the letter " $d$ " are the results with the consideration of damage). For all tested cases, the mechanical regularization length is kept to $\ell_{e}=20$ pixels

For any mesh size the same trends are observed, namely, the nominal DIC residual decreases with the addition of damaged elements. The enhancement is more pronounced for the larger regularization lengths. For instance, when the mesh size is equal to 8 pixels there is almost no difference between the two sets of calculations when $\ell_{a}=\ell_{b}=10$ pixels $(\omega=0.4 \%)$ while when $\ell_{a}=\ell_{b}=95$ pixels the residual level decreases from $2.4 \%$ (when no damage is applied) to $1.5 \%$ (when damaged is applied). This proves that the sought effect is achieved, namely, a better estimation of the gray level fields near laser shock edges (or large gradient zones) even if a large regularization length is applied.

\section{Experimental cases}

The aim of this section is to analyze two situations in which IR images were captured to measure thermal and displacement fields (Section 4.1) and to evaluate optical distortions (Section 4.2). In both cases, acquisition noise, which was not added in the synthetic case is present. In the second one, blur is also an issue that will be dealt with.

\subsection{Thermal shock assessment}

The investigation of thermal fatigue is the considered field of application of the present procedure. In the following, its feasibility is investigated. A new testing device was proposed [18], which allows for the use of optical methods. Only the images acquired by the IR camera will be analyzed.

Figure 9:

A pair of reference and deformed images and their initial difference (residuals) are shown in Figure 10. The deformed image (Figure 10(b)) undergoes 
large gray level variations in the impacted zone in addition to the motion induced by the thermomechanical behavior of the investigated material. If no gray level corrections are considered in the DIC formalism such images cannot be registered (see the initial residuals in Figure 10(c)).

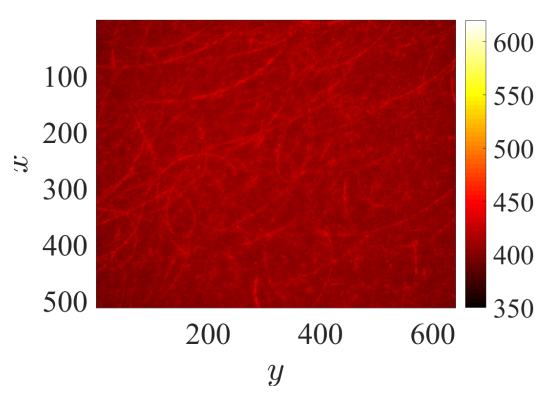

(a)

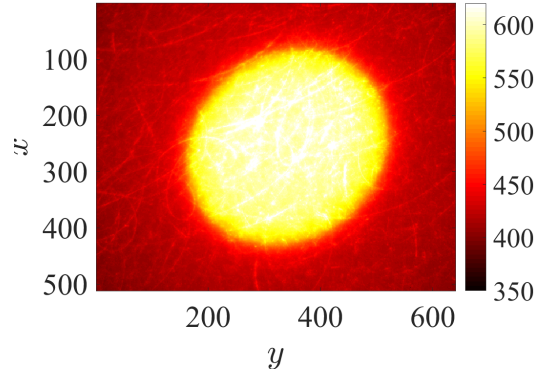

(b)

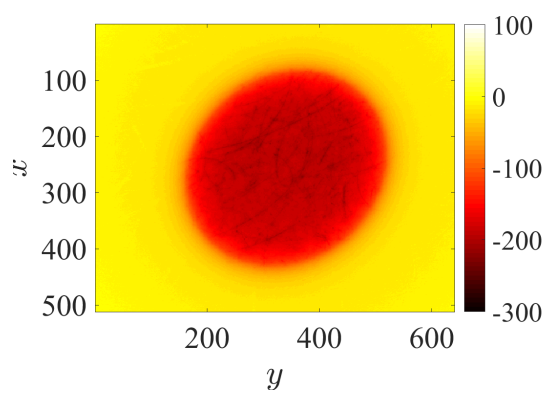

(c)

Figure 10: Reference (a) and deformed (b) IR images. (c) Initial correlation residuals (RMS: $120 \%$ of the dynamic range)

DIC accounting for regularized gray level corrections is run with a mesh size equal to 10 pixels, and regularization lengths of 20 pixels for both gray level and displacement fields. The estimated gray level corrections during the thermal shocks are shown in Figure 11. 


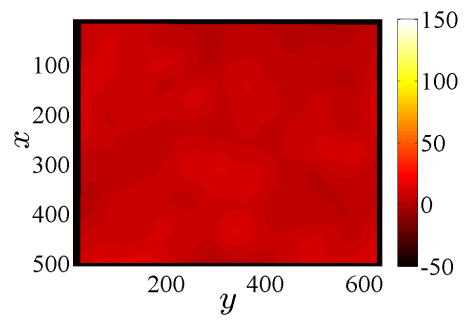

(a)

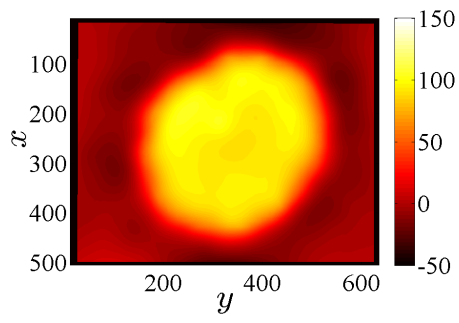

(c)

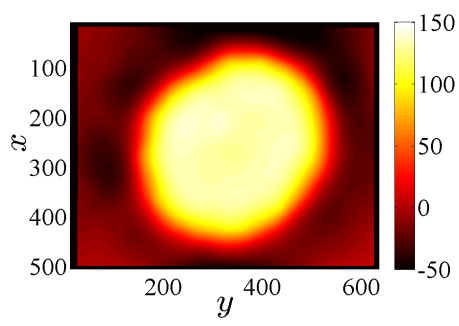

(e)

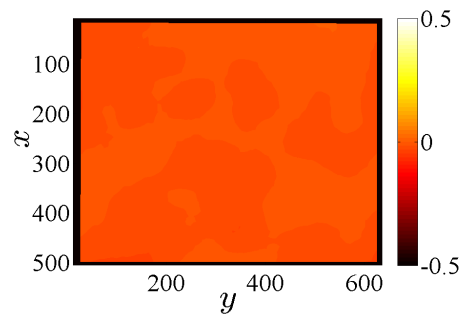

(b)

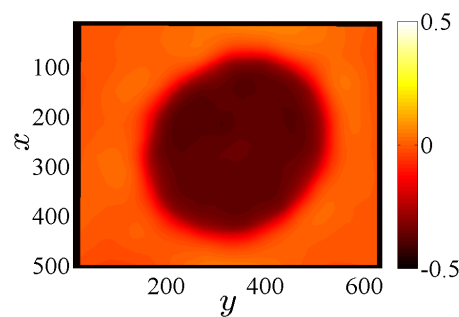

(d)

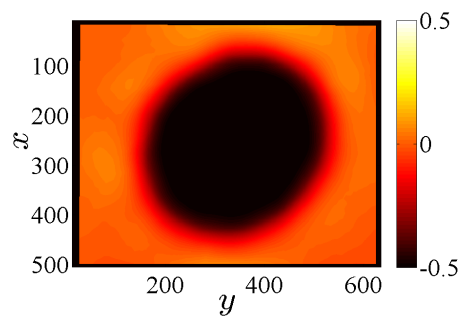

(f)

Figure 11: Gray level correction fields (left column: gray level brightness $a$, and right column contrast $b_{0}$ ) before (a-b) and during the thermal shock (c-d) when $t=30 \mathrm{~ms}$ and (e-f) $t=50 \mathrm{~ms}$

The 2D displacement fields correspond to a biaxial deformation induced by the laser shock (see Figure 12). Even though their dynamic range remains less than 0.5 pixel, they are well captured. 


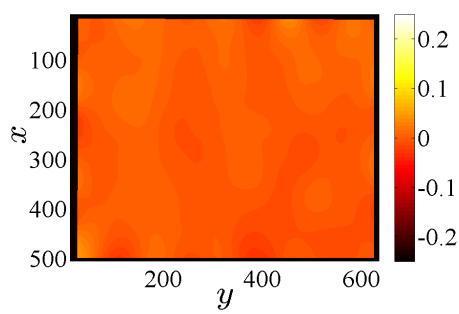

(a) $u_{x}$

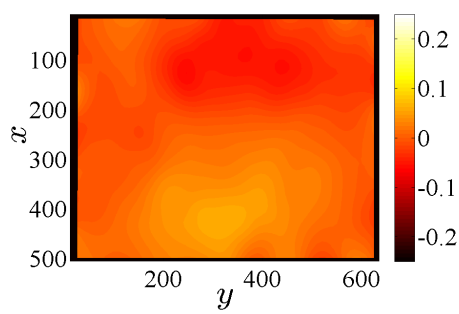

(c) $u_{x}$

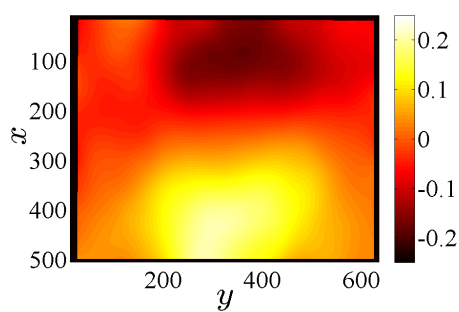

(e) $u_{x}$

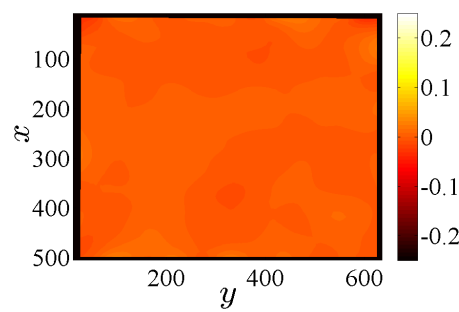

(b) $u_{y}$

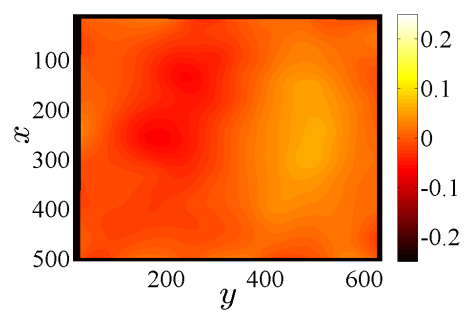

(d) $u_{y}$

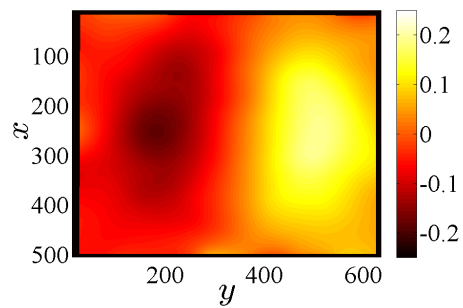

(f) $u_{y}$

Figure 12: Displacement fields expressed in pixel (1 pixel $\equiv 15 \mu \mathrm{m})$ before (a-b) and during the thermal shock (c-d) when $t=30 \mathrm{~ms}$ and (e-f) $t=50 \mathrm{~ms}$

At convergence, the residuals are reduced from $120 \%$ to less than $2 \%$ of the dynamic range when the images with the highest temperature levels are registered, thanks to the gray level corrections. Only 15 iterations are needed to reach convergence. The DIC residual maps are illustrated in Figure13. Except on the edge of the laser spot, the local levels are also very small. 


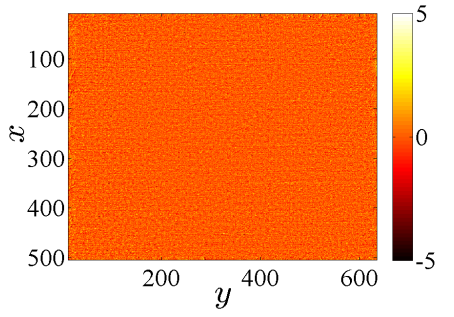

(a)

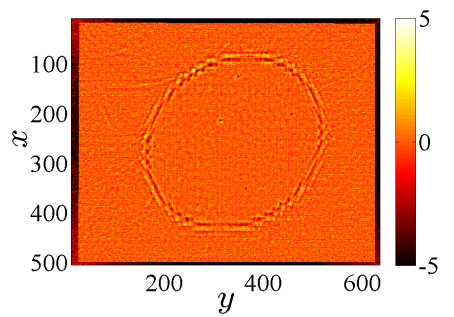

(b)

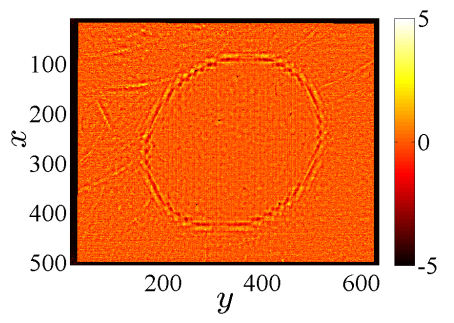

(c)

Figure 13: DIC residual maps (expressed in gray levels) before (a) and during the thermal shock (b) when $t=30 \mathrm{~ms}$ and (c) $t=50 \mathrm{~ms}$

As the element size is 10 pixels and the regularization lengths are set to 20 pixels the high temperature gradients near the laser spot are well captured. A ring can still be distinguished in the residual maps and has a thickness of about 10 pixels. Therefore the strategy with damage is not useful in that particular case as the regularization applied by the mesh cannot be reduced without considering smaller element sizes.

During the cooling stage the gray level fields are presenting less sharp distributions (Figure 14). The DIC residuals are uniform and do not reveal any ring. They started from $25 \%$ and were reduced to about $1 \%$ of the dynamic range at convergence. 


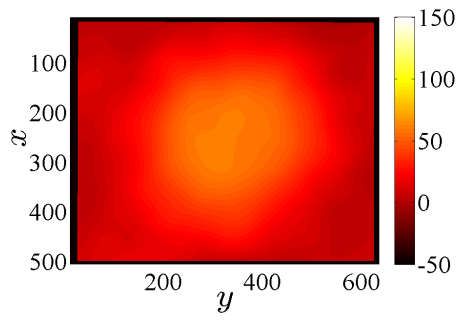

(a)

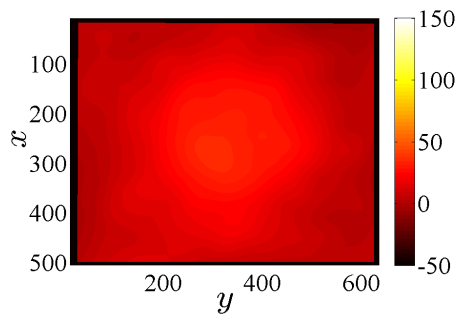

(c)

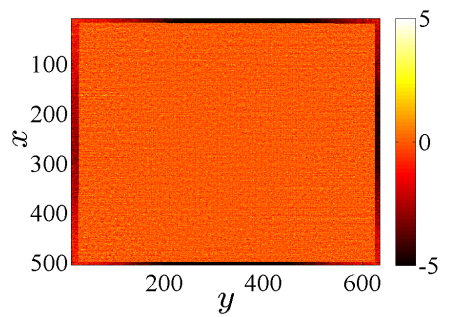

(b)

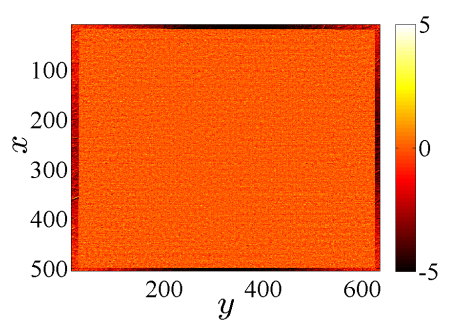

(d)

Figure 14: Brightness corrections (left) and DIC residual maps expressed in gray levels (right) after the thermal shock (a-b) when $t=75 \mathrm{~ms}$ and (c-d) $t=100 \mathrm{~ms}$

The displacement fields during the cooling phase of the thermal shock are shown in Figure 15. They are less pronounced during this phase in comparison with Figure 12. These fields can be further used to validate a numerical model and ensure that the selected constitutive parameters are well suited for the thermomechanical predictions [26]. 


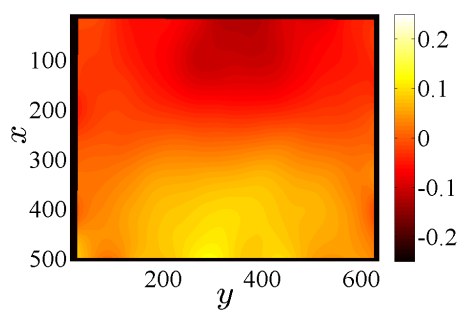

(a)

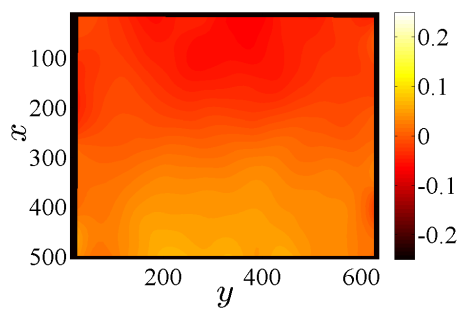

(c)

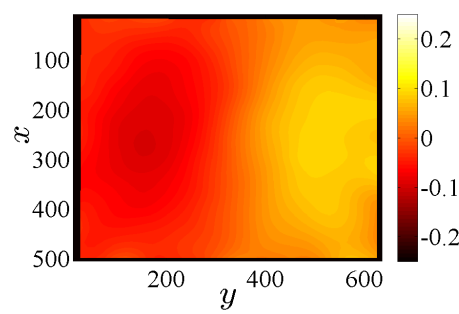

(b)

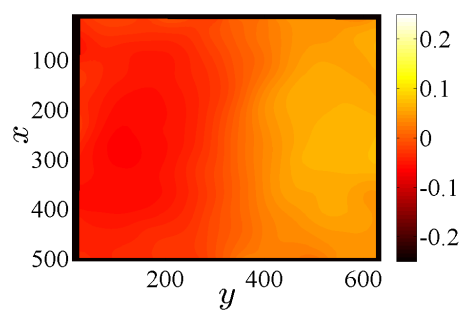

(d)

Figure 15: Displacement fields expressed in pixel $(1$ pixel $\equiv 15 \mu \mathrm{m})$ after the thermal shock (a-b) when $t=75 \mathrm{~ms}$ and (c-d) $t=100 \mathrm{~ms}$

Up to now only brightness and contrast fields have been discussed as in the case of laser shocks or more generally thermomechanical tests implying neither out-of-plane motions nor blur (from the initial to deformed states). The next subsection illustrates the ability of the present DIC formulation to account for blur corrections for the particular case of calibration, which has been discussed in Ref. [11.

\subsection{Optical lens distortion}

One of the camera calibration step consists in correcting optical lens distortion. This can be performed by using a patterned calibration target, which is pictured by the imaging system [11. A reference image is generated numerically (i.e., synthetic target) and hence is free from any deformation due to lens distortion. The displacement fields obtained by registering the pictured and synthetic target images are interpreted as distortions (after subtraction of rigid body motions for positioning and scaling). Two strategies can be applied. The first one is to use an integrated DIC algorithm that directly provides the parameters of distortion models (such as Zernike polynomials [12, 13]). The second approach is to use a generic DIC algorithm and either low-pass filter the measured displacement or project it onto a suited basis (such as generic distortion fields [5]). In both cases, it is necessary to consider gray level variations between the reference and deformed images, which are from different origins, 
see Figure 16). In the case of IR cameras for instance the frames are often blurred in the edges (Figure 16a).

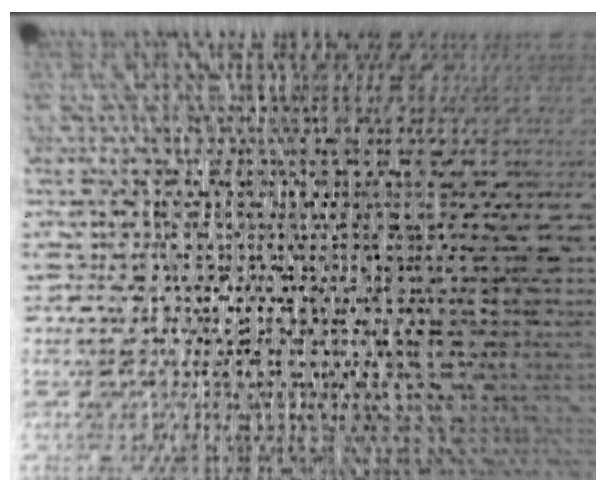

(a)

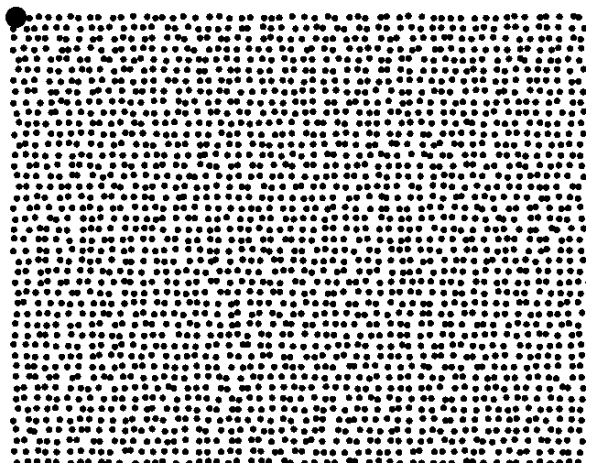

(b)

Figure 16: (a) Acquired (with IR camera) and (b) reference images of a calibration target

The proposed formulation (1) is well-suited for such effects. Only two blur kernels are considered in the sequel. The contrast, brightness and blur fields are regularized (i.e., $\ell_{a}=\ell_{b}=12$ pixels).

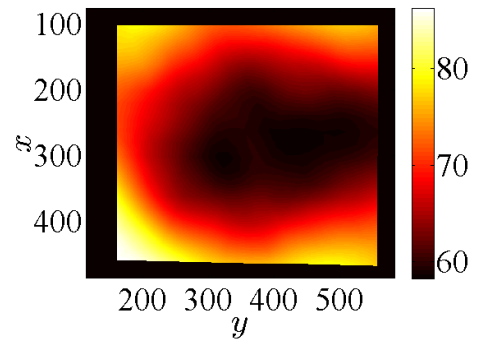

(a)

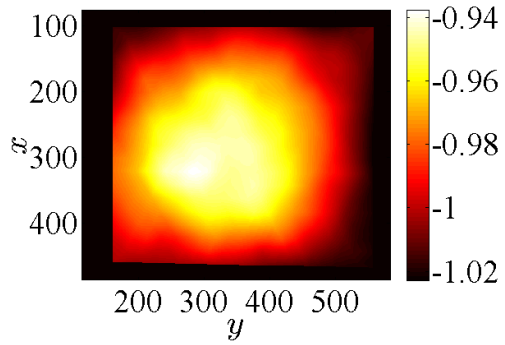

(b)

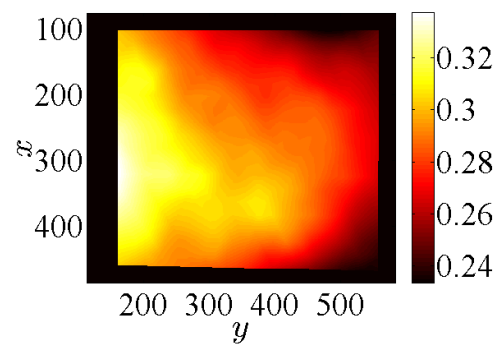

(c)

Figure 17: Gray level correction fields when binary and IR frames are registered. (a) Gray level brightness, (b) contrast and (c) weight applied to blur corrections 
Figure 17 shows the gray level correction fields. The elliptical shape of the fields corresponds to a slight vignetting effect. This is consistent with the results reported in Ref. [11. It is noteworthy that significant blur is present, and is not uniform over the image. To highlight the relevance of blur, Figure 18 shows the residual fields obtained when blur corrections are or are not considered. The significant reduction in residual magnitude, from $\omega=10 \%$ to $5 \%$, shows the importance of accounting for blur. The same trend was observed in Ref. [11.

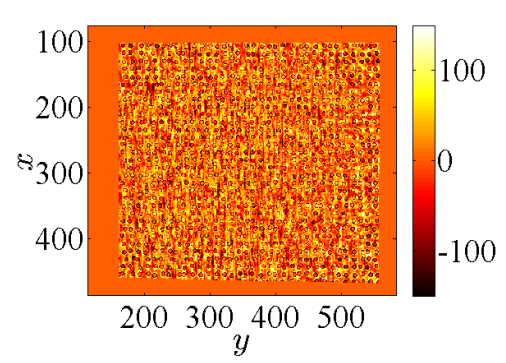

(a)

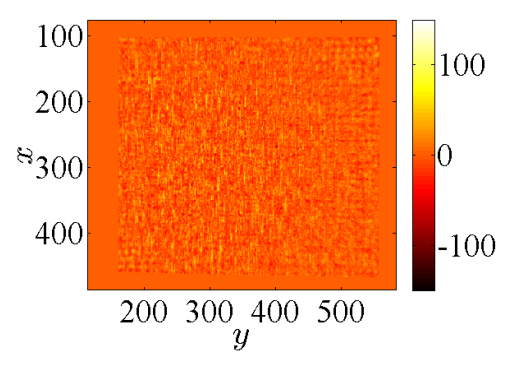

(b)

Figure 18: DIC residual maps (expressed in gray levels) obtained either (a) without or (b) with blur corrections

The distortion displacement field is shown in Figure 19. In Ref. 11 FEDIC and integrated DIC were compared and the RMS gap was of the of order 0.1 pixel between the two methods. In the present case the same level is observed.

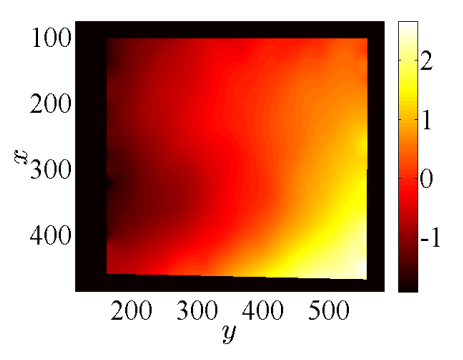

(a) $u_{x}$

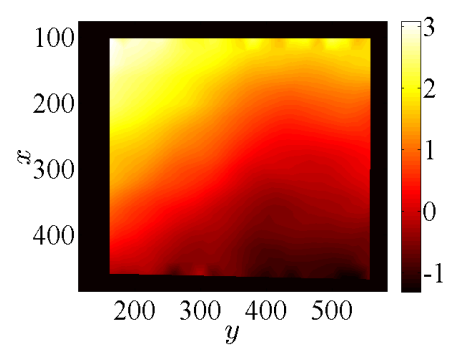

(b) $u_{y}$

Figure 19: Displacement fields when binary and IR frames are registered

\section{Conclusions}

A generalized formulation for global DIC purposes is proposed in which the hypothesis of gray level conservation is relaxed. Brightness, contrast and blur corrections are decomposed onto a finite element mesh, the same as that chosen 
for the kinematic basis. In order to preserve good conditioning of the problem, regularization is proposed so that these fields may be less dependent on the mesh (i.e., independent if regularization lengths are large enough).

A synthetic test case, which is designed to mimic a real experimental situation, was tested to probe the sensitivity and reliability of such an approach for a difficult configuration where sharp variations of the correction field are present. The interplay of regularization length scales and mesh size was considered and shown to impact convergence speed as well as the level of residuals. It is also shown that although the gray level corrections are essential for a successful DIC, the details of gray level correction has a negligible influence on the displacement field (i.e., the root mean square error being of the order of 0.03-0.04 pixel), thereby showing that the proposed algorithm is very robust. A strategy aiming at adapting locally the regularization length is proposed and shown to be an effective way of handling situations where the regularization assumptions are expected to be violated.

Application to a real experimental case allowed the displacement field to be measured with an IR camera from the observation of the surface that is heated by a laser beam. For this application, the gray level variations due to the temperature field are much larger than the texture modulation due to the kinematic field, and yet the displacement could be estimated and favorably compared with a model. After a suitable calibration, the gray level corrections also provide the surface temperature field. Further comparisons 26 can be performed with that single IR camera.

The same methodology was also shown to be applicable to distortion measurements from the registration between a binary model and its IR image. The gray level corrections allow vignetting and blur to be corrected as can be judged from the (low) residual fields.

\section{References}

[1] T. C. Chu, W. F. Ranson, and M. A. Sutton, "Applications of digital-imagecorrelation techniques to experimental mechanics," Experimental Mechanics, vol. 25, no. 3, pp. 232-244, 1985.

[2] J. S. Lyons, J. Liu, and M. A. Sutton, "High-temperature deformation measurements using digital-image correlation," Experimental Mechanics, vol. 36, no. 1, pp. 64-70, 1996.

[3] B. Wattrisse, A. Chrysochoos, J.-M. Muracciole, and M. Némoz-Gaillard, "Analysis of strain localization during tensile tests by digital image correlation," Experimental Mechanics, vol. 41, no. 1, pp. 29-39, 2001.

[4] S. Avril, F. Pierron, M. A. Sutton, and J. Yan, "Identification of elastovisco-plastic parameters and characterization of Lüders behavior using digital image correlation and the virtual fields method," Mechanics of Materials, vol. 40, pp. 729-742, Sept. 2008. 
[5] M. A. Sutton, J. Orteu, and H. W. Schreier, Image Correlation for Shape, Motion and Deformation Measurements. Springer, 2009.

[6] F. Hild and S. Roux, "Digital Image Correlation," in Optical Methods for Solid Mechanics : A Full-Field Approach, Wiley-VCH, Berlin (Germany). P. Rastogi and Editor E. Hack (Edts.), 2012.

[7] J. Réthoré, T. Elguedj, M. Coret, P. Chaudet, A. Combescure, "Robust identification of elasto-plastic constitutive law parameters from digital images using 3D kinematics," International Journal of Solids and Structures, vol. 50, no. 1, pp. 73-85, 2013.

[8] W. Tong, "Formulation of Lucas-Kanade Digital Image Correlation Algorithms for Non-contact Deformation Measurements: A Review," Strain, vol. 49, no. 4, pp. 313-334, 2013.

[9] B. Pan, H. M. Xie and Z. Y. Wang, "Equivalence of Digital Image Correlation Criteria for Pattern Matching," Applied Optics, vol. 49, no. 28, pp. 5501-5509, 2010.

[10] B. D. Lucas and T. Kanade, "An Iterative Image Registration Technique with an Application to Stereo Vision," in Proceedings of DARPA Image Understanding Workshop, pp. 121-130, 1981.

[11] A. Charbal, J.-E. Dufour, A. Guery, F. Hild, S. Roux, L. Vincent, and M. Poncelet, "Integrated Digital Image Correlation considering gray level and blur variations: Application to distortion measurements of IR camera," Optics and Lasers in Engineering, vol. 78, pp. 75-85, 2016.

[12] D. Brown, "Decentering distortion of lenses," Photogrammetric Engineering, vol. 56, pp. 444-462, 1966.

[13] D. Brown, "Close-range camera calibration," Photogrammetric Engineering, vol. 37, no. 8, pp. 855-866, 1971.

[14] A. Maynadier, M. Poncelet, K. Lavernhe-Taillard, and S. Roux, "One-shot measurement of thermal and kinematic fields: Infra-Red Image Correlation (IRIC)" Experimental Mechanics, vol. 52, no. 3, pp. 241-255, 2011.

[15] R. Szeliski, Computer Vision: Algorithms and Applications. Springer, 2010.

[16] M. Poncelet and H. Leclerc, "A Digital Image Correlation Algorithm with Light Reflection Compensation," Experimental Mechanics, vol. 55, no. 7, pp. 1317-1327, 2015.

[17] A. Charbal, J. E. Dufour, F. Hild, S. Roux, L. Vincent, and M. Poncelet, "Hybrid stereocorrelation using infrared and visible light cameras," Experimental Mechanics, vol. 56, no. 5, pp. 845-860, 2016.

[18] L. Vincent, M. Poncelet, S. Roux, F. Hild, and D. Farcage, "Experimental Facility for High Cycle Thermal Fatigue Tests Using Laser Shocks," Procedia Engineering, vol. 66, pp. 669-675, 2013. 
[19] S. Utz, E. Soppa, K. Christopher, X. Schuler, and H. Silcher, "Thermal and mechanical fatigue loading - Mechanisms of crack initiation and crack growth," (Anaheim, California, USA), p. 10, 2014.

[20] A. N. Tikhonov and V. Y. Arsenin, Solutions of Ill-posed problems. New York: J. Wiley, 1977.

[21] Z. Tomicevic, F. Hild, and S. Roux, "Mechanics-aided digital image correlation," The Journal of Strain Analysis for Engineering Design, vol. 48, no. 5, pp. 330-343, 2013.

[22] J. Réthoré, S. Roux, and F. Hild, "An extended and integrated digital image correlation technique applied to the analysis of fractured samples," European Journal of Computational Mechanics, vol. 18, pp. 285-306, 2009.

[23] G. Broggiato, L. Casarotto, Z. Del Prete, and D. Maccarrone, "Full-field strain rate measurement by white-light speckle image correlation," Strain, vol. 45, no. 4, pp. 364-372, 2009.

[24] G. Besnard, H. Leclerc, S. Roux, and F. Hild, "Analysis of Image Series through Digital Image Correlation," Journal of Strain Analysis, vol. 47, no. 4, pp. 214-228, 2012.

[25] A. Charbal, S. Roux, F. Hild, and Vincent, "Spatiotemporal regularization for digital image correlation: Application to infrared camera frames," submitted for publication, 2017.

[26] A. Charbal, L. Vincent, F. Hild, M. Poncelet, J.-E. Dufour, S. Roux, and D. Farcage, "Characterization of temperature and strain fields during cyclic laser shocks," Quantitative InfraRed Thermography Journal, vol. 13, no. 1, pp. 1-18, 2016.

[27] J. Lemaitre, A Course on Damage Mechanics, Springer-Verlag, Berlin (Germany), 1992. 